D.O.I.:10.3895/S1808-04482012000100005

\title{
APLICAÇÃO DO PROKNOW-C PARA SELEÇÃO DE UM PORTIFÓLIO BIBLIOGRÁFICO E ANÁLISE BIBLIOMÉTRICA SOBRE AVALIAÇÃO DE DESEMPENHO DA GESTÃO DO CONHECIMENTO
}

\section{APPLICATION OF PROKNOW-C TO SELECT BIBLIOGRAPHY PORTFOLIO AND BIBLIOMETRIC ANALYSIS ABOUT PERFORMANCE EVALUATION OF KNOWLEDGE MANAGEMENT}

\author{
Lílian de Oliveira Vilela ${ }^{1}$; \\ ${ }^{1}$ Universidade Federal de Santa Catarina - UFSC - Florianópolis - Brasil \\ lilian.oliveira.vilela@gmail.com
}

\begin{abstract}
Resumo
O objetivo do estudo foi aplicar o método ProKnow-C, desenvolvido pelo Laboratório MCDA da Universidade Federal de Santa Catarina, para pesquisar e analisar quantitativamente artigos de prestígio científico e relevantes para o tema Gestão do Conhecimento sob a ótica da avaliação de desempenho. $O$ método utilizado trata-se da sistematização do processo de busca e seleção de artigos proposta pelo ProKnow-C, fazendo uso de técnicas de análise bibliométrica e também considerando o que o pesquisador julga relevante para o tema de pesquisa.Como principal resultado tem-se a validação do método utilizado para construção do conhecimento sobre assunto pesquisado. Não menos importante, a aplicação do método permitiu obter um portifólio bibliográfico relevante e de prestígio científico. Detalhes, como os principais autores e principais palavras-chave empregadas para o tema, são de interesse para pesquisadores da área. Futuramente, o resultado obtido nesta pesquisa através da aplicação do método pode contribuir para estudos cujo interesse resida na evolução, no tempo, do tema: avaliação de desempenho da gestão do conhecimento.
\end{abstract}

Palavras-chave: bibliometria; revisão da literatura; avaliação de desempenho; gestão do conhecimento.

\section{Introdução}

Pesquisas fundamentadas em engenharia de produção costumam iniciar com a seleção de documentos de prestígio científico para a composição da fundamentação teórica e identificação das lacunas na literatura (CAUCHICK et al., 2010). Diante da quantidade de artigos científicos existentes nas bases de dados atualmente, uma das dificuldades do pesquisador é encontrar aqueles que são de maior relevância para o tema que se deseja pesquisar.

Sabendo da necessidade de justificar a escolha dos conceitos básicos utilizados para fundamentar novas pesquisas aliada a dificuldade de selecionar artigos de prestígio científico é que se proprõem, nesse estudo, aplicar o método ProKnow-C, Knowledge Development Process- 
Constructivist (Ensslin, et al., 2010), da Universidade Federal de Santa Catarina. A aplicação tem, por sua vez, o objetivo de selecionar e analisar quantitavidamente um portfólio bibliográfico sob o tema: avaliação de desempenho da gestão do conhecimento (GC). A escolha do ProKnow-se como meio de intervenção nesse processo se justifica na simplicidade e sistematização da atividade para a construção do conhecimento necessário para iniciar uma pesquisa, conforme conclusão de autores como Afonso et al. (2012), e Tasca et al. (2010).

O interesse no tema GC reside no seu reconhecimento, cada vez maior, como recurso significativo na busca de vantagem competitiva pelas organizações (DAVENPORT et al., 1998; GLOET; TERZIOVSKI, 2004; PABLO, 2004).

Diante das dúvidas existentes na literatura em relação a como realizar a avaliação de desempenho da gestão do conhecimento a fim de verificar sua efetividade enquanto processo de maximização dos retornos financeiros, é que surge a curiosidade em aprofundar as pesquisas nesse tema.

Para buscar na literatura as iniciativas existentes na experimentação de métricas para avaliar a gestão do conhecimento nas organizações e, conhecendo a dificuldade de filtrar o material acadêmico disponível é que se propõem como objetivo geral desse trabalho: aplicar o método ProKnow-C para aprofundar o conhecimento sobre o tema definido. Como objetivos específicos, etapas para alcançar o objetivo geral, tem-se (i) selecionar artigos em periódicos qualificados, de reconhecimento científico e alinhados com o tema de pesquisa. (ii) selecionar palavras-chave de pesquisa; (iii) selecionar as bases de dados alinhadas com o tema de pesquisa; (iv) selecionar os artigos alinhados com o tema de pesquisa e (v) identificar os artigos relevantes da amostra selecionada (vi) validar o método utilizado como instrumento de construção do conhecimento.

A justificativa desse estudo está não só no crescente interesse pelo tema "avaliação da gestão do conhecimento", mas, principalmente, na dificuldade existente para qualquer pesquisador em encontrar um método que sistematize a atividade de pesquisa nas bases de dados para busca de material de reconhecimento científico.

A relevância da pesquisa está na ilustração do método ProKnow-C, capaz de contrubuir com pesquisadores de diversas áreas na seleção de portifólio bibliográfico e análise bibliométrica.

Como resultado direto tem-se a validação do método como instrumento de auxílio na construção do conhecimento através da obtenção de avaliações quantitativas do portfólio de artigos selecionados assim como o conhecimento base para iniciar uma pesquisa sobre o tema de avaliação da gestão do conhecimento.

A pesquisa está estruturada, no item 2, com um breve referencial teórico dos temas abordados na pesquisa: avaliação de desempenho da gestão do conhecimento e seleção e avaliação de portifólio bibliofráfico. No item 3 tem-se os procedimentos metodológicos aplicados. No item 4 
verifica-se os resultados obtidos e, por fim, as considerações finais podem ser encontradas no item 5.

\section{Referencial teórico}

Diante da problemática geral da pesquisa é necessário elucidar os conceitos utilizado aqui tanto para a definição do que seja avaliação de desempenho da gestão do conhencimento no contexto das organizações como também para os conceitos básicos de pesquisa e análise de portifólio bibliográfico. Isso se faz necessário uma vez que o processo de seleção de documentos de interesse está relacionado ao conceito que o pesquisador tem ou deseja ter do tema de pesquisa.

No contexto organizacional, a GC é interpretada por Chourides et al. (2003) como o processo de gerir a aquisição de conhecimento. Terra (2005) define: "a GC se ocupa dos processos gerenciais e de infraestrutura física e digital que facilitam, favorecem e estimulam os processos de criação, compartilhamento e uso dos conhecimentos individuais e coletivos".

Para Bose (2004), a razão de gerenciar e fazer crescer o conhecimento das empresas é a maximização dos retornos financeiros e, para obter tal vantagem, é preciso ser capaz de medir o investimento e o benefício deste investimento com determinada frequência, ou seja, é necessário avaliar o desempenho desse processo (CHOURIDES; LONGBOTTOM; MURPHY, 2003). No entanto, para o mesmo autor, através de uma revisão da literatura sobre métricas da GC, medir os benefícios da GC não é uma tarefa simples. Talvez, por esse motivo, Chen e Chen (2006) identificam poucos estudos empíricos na literatura dedicados a avaliar o desempenho GC nas organizações, como é o caso do estudo de Spicer e Sadler-Smith (2006).

De posse das dúvidas e/ou lacunas na literatura sobre o tema que se deseja pesquisar é que o pesquisador busca informações nas tradicionais bases de dados bibliográficas. Como base de dados entende-se, aqui, fontes bibliográficas de trabalhos como artigos, jornais, teses, livros, relatórios técnicos, textos de conferências, etc.

O sucesso ou fracasso de uma pesquisa depende, entre outros fatores, da definição da estratégia de busca de informação nas bases de dados que pode ser: via palavras-chave, autores, assuntos, etc. Atualmente, dispõem-se de ferramentas cada vez mais eficientes para a busca de informações devido ao avanço tecnológico ter permitido uma base de dados eletrônica, assim como ferramentas adequadas de busca nesse meio (TASCA et al. 2010).

Após definidas as estratégias de busca de estudos científicos, vem a árdua tarefa de selecionar o material obtido. A fim de facilitar esse processo, há tempos os pesquisadores utilizamse dos "estudos métricos" para avaliar a informação produzida com base em recursos quantitativos como ferramenta de análise, como por exemplo, o estudo de Narin de 1975 (NARIN, 1976). Oliveira e Gracio (2011) explicam que os estudos métricos desenvolveram-se a partir da 
blibliometria e outras abordagens como a cientometria, webometria, informetria, patentometria que envolvem um amplo conjunto de indicadores bibliométricos

O objeto de estudo da bibliometrica consiste nas análises da produção científica, das citações e dos conteúdos produzidos (ARAUJO, 2006; MORETI; CAMPANARIO, 2009) apresentando uma maneira quantitativa de avaliar sua relevância através de indicadores para nortear

o processo. Vários são os indicadores encontrados na litratura sobre bibliometria. Moreti e Campanario (2009) consideram de grande utilidade, por exemplo, para a compreenção da produtividade e do avanço do conhecimento gerado sobre um tema, verificar quais são os autores e centros de pesquisas mais produtivos e que mais contribuem para o desenvolvimento do tema de estudo. A justificativa, para os autores, está no fato de que pode-se estimar a consolidação de uma área de estudo quanto maior for a a produção de autores nessa área dentro de um determinado período de tempo.

\section{Procedimentos metodológicos}

Para o alcance do objetivo da pesquisa, aplicou-se 2 das 4 principais etapas do processo ProKnow-C de Ensslin et al. (2010), ilustradas na Figura 1 para o tema avaliação de desempenho da gestão do conhecimento.

Figura 1: Etapas do processo ProKnow-C

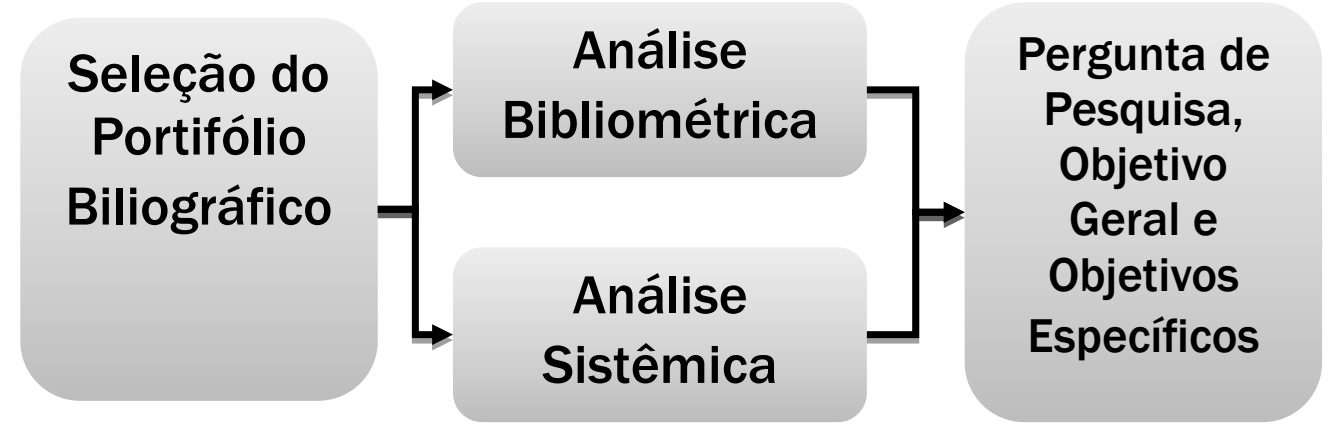

Fonte: Adaptado de Ensslin et al. (2010)

A seguir, a descrição das etapas do processo ProKnow-C que foram aplicadas em Março de 2011 para obtenção dos resultados descritos no item 4:

a) Seleção de Portfólio Bibliográfico: consistiu na busca de material científico nas bases de dados de maneira sistematizada. Inicialmente, definiu-se as palavras-chave sobre o tema de pesquisa na lingua inglesa separando-as em 2 eixos de pesquisa. Em seguida, foram selecionadas as bases de dados no Portal Capes (CAPES) que maior relação possuíam com o tema de pesquisa de acordo com os conceitos dos autores. Foram encontradas cerca de 15 bases e mais de 400.000 documentos relacionados à GC. Após a busca de artigos científicos escritos na lingua inglesa, o 
processo apresenta uma série de filtros para gerar o portfólio de artigos mais relevantes para o tema. Esses filtros se referem à redundância (artigos repetidos no portifólio), alinhamento pelo título e reconhecimento científico. Como resultado, foram obtidos artigos "disponíveis” (artigos cujo acesso não requer pagamentos), alinhados com o tema e reconhecidos cientificamente;

b) Análise Bibliométrica: evidenciou-se quantitativamente os dados estatísticos do conjunto de artigos obtido na etapa (a), realizado por meio da contagem de parâmetros como: publicações, autores, citações, periódicos, bases de dados. Assim, foram identificados três grupos de indicadores: o primeiro está relacionado com os artigos do portfólio, diretamente. O segundo, com as referências existentes nos artigos do portfólio e, por fim, o terceiro grupo de indicadores quantifica a relação entre os dados do primeiro grupo com os do segundo.

\section{Apresentação, análise e discução dos resultados obtidos}

Essa seção tem o objetivo de descrever processo de seleção de portfólio e análise bibliométrica ilustrando a utilização do método ProKnow-C para o tema: avaliação de desempenho da gestão do conhecimento.

\subsection{Seleção de portfólio bibliográfico}

\subsubsection{Seleção do banco de artigos brutos}

A seleção do banco de artigos brutos consiste na busca dos artigos sobre o tema selecionado. No método ProKnow-C são considerados somente artigos na lingua inglesa publicados em periódicos qualificados. Não são consideradas teses, dissertações ou artigos de congressos.

- Definir as palavra-chaves (PC): para definição das PC foi necessário identificar, inicialmente, os eixos de pesquisa. Para cada eixo, foram selecionadas as palavras relacionadas com o assunto. As PCs são geradas a partir da combinação das palavras de cada eixo de pesquisa conforme ilustrado na Figura 2.

Figura 2: Eixos de pesquisa e palavras-chave

\begin{tabular}{|l|l|}
\hline EIXO I: AVALIAÇÃO DE & EIXO II: GESTÃO DO \\
DESEMPENHO & CONHECIMENTO \\
Performance Measurement & Organizational Learning \\
Performance Evaluation & Management Information \\
Performance Assessment & Knowledge Management \\
Performance Appraisal & Creation of Knowledge \\
\hline
\end{tabular}

Fonte: Resultado da pesquisa (2011)

- Definir Banco de Dados: para a seleção das bases de dados foram utilizadas as informações sobre as bases no Portal de Periódicos (CAPES) e selecionadas as bases de maior 
aderência com o assunto da pesquisa (ISI Web of Knowledge, SCOPUS, IEEE, Wilson, Science Direct, Emerald, SCIRUS). Em seguida, fez-se o alinhamento das PCs buscando o número de artigos encontrados em cada base por PC. Como critério de busca nas ferramentas das próprias bases de dados, definiu-se procurar por artigos que tivessem as PCs em seus títulos, resumos ou palavras-chave. Outro critério utilizado foi o de procurar documentos recentes, isto é, dos últimos dez anos. Subjetivamente foi fixada a representatividade de $90 \%$ para seleção final das bases de pesquisa. Nesse caso, permaneceu-se com as bases relacionadas na Figura 3:

Figura 3: Bases de dados e número de artigos encontrados

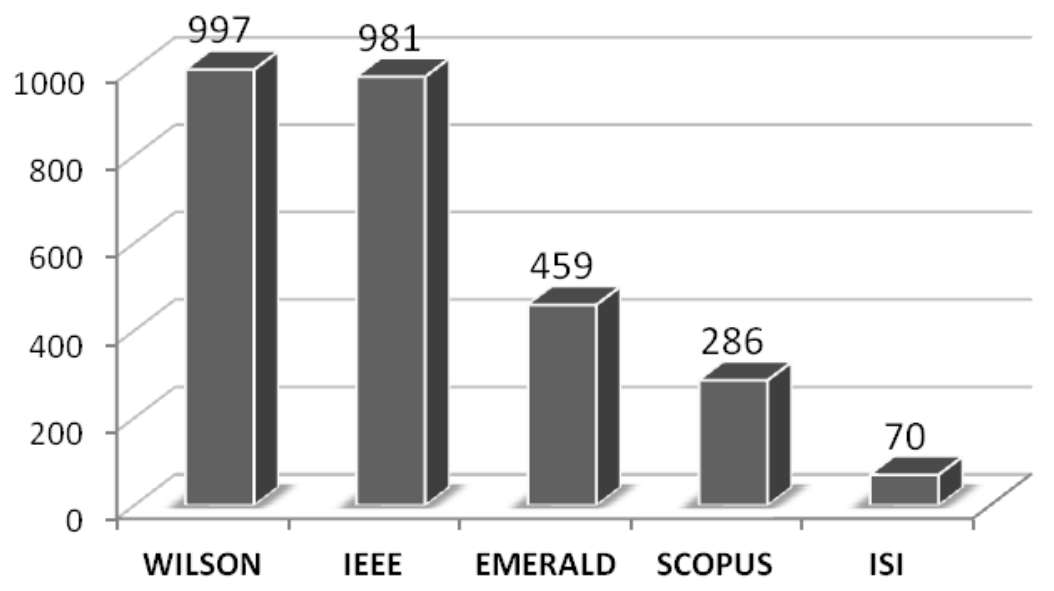

Fonte: Resultado da pesquisa (2011)

- Teste de aderência das palavras-chave: nesta fase do processo é necessário verificar se as palavras-chave inicialmente selecionadas são mesmo as que melhor buscam artigos relacionados com o tema. Para isso, o método ProKnow-C sugere que sejam selecionados dois artigos do portfólio bruto obtido cujo título sugira a adequação do documento com o assunto de pesquisa. Depois disso, analisar as PCs utilizadas pelos autores desses artigos a fim de verificar a aderência às PCs utilizadas na busca do banco de dados. Caso não haja alinhamento das palavras ou existam outras PCs mais adequadas, recomenda-se voltar à primeira etapa: seleção das palavras-chave e refazer o banco de artigos brutos. Caso seja verificado o alinhamento, o resultado do banco já é o resultado desse sub-processo: o banco de artigos bruto (BAB).

No caso do presente estudo, as PCs se mostraram muito bem alinhadas. Foram testadas outras palavras encontradas nos 2 artigos de referência: "intelectual capital", "total quality" e "continuous improvement". No entanto, para a palavra capital intelectual combinada com as quatro palavras do eixo de avaliação de desempenho, não se obteve um resultado significativo de artigos. Por outro lado, com as palavras melhoria contínua e qualidade total, o portfólio de artigos aumentou significamente, porém, pelos títulos, foi possível observar que grande parte deles não estava totalmente alinhado à gestão do conhecimento. 
O resultado da etapa de seleção dos artigos brutos foi, portanto, as 16 combinações de palavras dos dois eixos de pesquisa definidos, isto é, foram obtidas 16 palavras-chave (PC). Também foi obtido o banco de dados, isto é, as bases de dados para a busca de artigos. Por fim, o banco de artigos brutos com 2.723 artigos reconhecidos cientificamente.

\subsubsection{Filtragem do banco de artigos brutos}

A Filtragem do BAB consiste na seleção dos artigos disponíveis, de maior relação com o tema de pesquisa e de relevância significativa. A seguir, os filtros pelos quais passarão os artigos do $\mathrm{BAB}$ para dar origem ao portfólio bibliográfico final.

- Filtro do BAB quanto à redundância: primeiramente, é necessário eliminar do BAB os artigos repetidos, uma vez que um mesmo documento pode existir em mais de uma base de dados. Para isso, pode-se utilizar a ferramenta EndNote, software de dados para o qual é possível exportar as informações do artigos selecionados nas bases (título, resumo, autores, URL, DOI). Esse software também oferece uma ferramenta de identificação dos artigos duplicados. Assim, para essa pesquisa, 51\% dos artigos estavam duplicados. Com a aplicação desse filtro, o BAB passou de 2.723 para 1.339 artigos brutos não repetidos;

- Filtro do BAB não repetidos quanto ao alinhamento do título: nessa etapa foi realizada a leitura integral dos títulos dos 1.339 artigos e excluídos do banco aqueles cujo título não tivesse relação com a avaliação de desempenho da gestão do conhecimento. Dessa forma, foram eliminados 1.158 artigos não relacionados com o tema. O BAB de artigos não repetidos, então, ficou com 181 artigos, apenas 13,5\% do total;

- Filtro do BAB não repetidos quanto ao reconhecimento científico: nesta fase é necessário determinar o número de citações de cada artigo da base. Para isso, foram utilizadas as informações disponíveis no Google Acadêmico (GOOGLE ACADÊMICO) que, para cada artigo, traz o número de vezes em que ele foi citado em outros documentos. Assim, define-se, subjetivamente, a representatividade, isto é, o percentual acumulado que os artigos mais citados representam. Para o presente estudo, optou-se por selecionar os 63 artigos responsáveis por 90\% das citações dos 181 artigos do BAB não repetidos e alinhados quanto ao título. Nesse caso, foram selecionados artigos com até 14 citações. Utilizando a classificação ABC, os $90 \%$ representam o grupo de artigos A e B conforme ilustrado na Figura 4. A opção por $90 \%$ como fator de representatividade é decorrente do número de artigos resultantes. Percentuais abaixo de $90 \%$ trariam, além de um número baixo de artigos para o próximo filtro, a possibilidade de descartar artigos com número de citações razoável. Como resultado, tem-se uma base com os 63 artigos mais citados e outra base com 118 artigos cuja relevância ainda deve ser verificada. Isso é necessário uma vez que artigos muito recentes ainda não 
possuem um número de citações que seja representativa para enquadrá-lo na base dos mais citados;

- Seleção da base de artigos não repetidos, título alinhado e com reconhecimento científico: essa base, com 63 artigos, passa, agora, por filtro de alinhamento do resumo com o tema de pesquisa. Nesse caso, a seleção do que é relevante ou não fica depende da escolha do pesquisador, do que ele julga relevante para o tema. Assim, foram selecionados 26 artigos cujo assunto se referia à determinação de métricas para GC e estudos empíricos de avaliação de performance da GC;

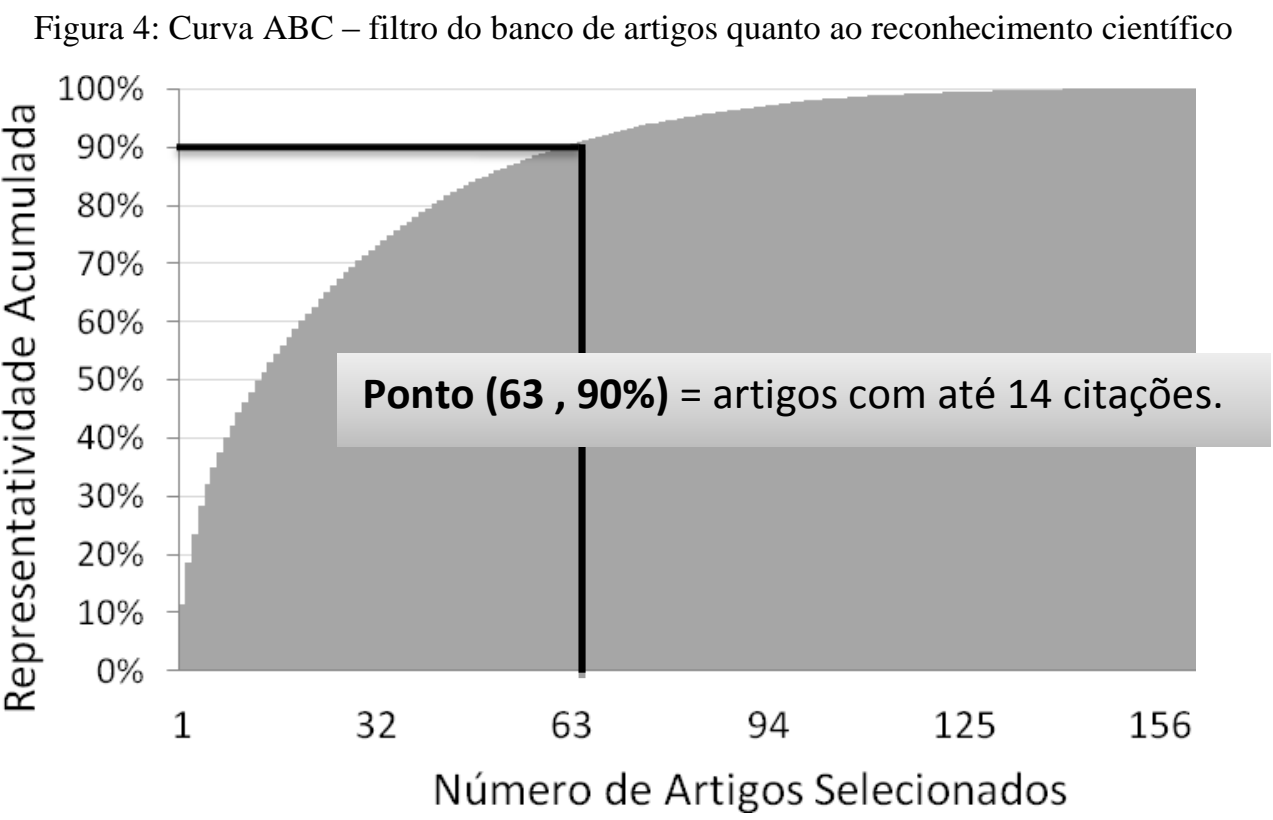

Fonte: Resultado da pesquisa (2011)

- Seleção da base de artigos não repetidos, título alinhado e com reconhecimento científico não confirmado: a identificação dos 118 artigos restantes como reconhecidos ou não é realizada através da leitura dos resumos dos artigos com até 2 anos de publicação. Esse critério, como dito anteriormente, é necessário uma vez que artigos muito recentes não possuem ou possuem poucas citações. Dos 118 artigos dessa base, 32 possuíam menos de 2 anos da data de publicação. Dos 86 restantes, o método ProKnow-C sugere que sejam avaliados os autores, isto é, se algum dos artigos possui o mesmo autor dos artigos com reconhecimento científico. No caso dessa pesquisa, apenas 3 artigos eram dos mesmos autores dos 63 reconhecidos cientificamente. Assim, faz-se a leitura dos resumos dos 32 atigos recentes mais 3 artigos de autores reconhecidos. O resultado obtido foi o de 8 artigos alinhados com o tema de pesquisa (mesmo critério adotado para a base anterior);

- Filtro com relação à disponibilidade: nesta fase, a partir da base de 26 mais 8 artigos alinhados e reconhecidos cientificamente, é necessário verificar sua disponibilidade, isto é, documentos cujo acesso não requer aquisição e está isento de taxas ou filiações. Dos 34 artigos selecionados, 12 não estavam disponíveis;

- Filtro com relação ao alinhamento do texto completo: etapa final do processo de seleção, 
esse filtro consiste na leitura dos artigos alinhados e disponíveis. Para o presente estudo, dos 22 artigos restantes, 11 não estavam totalmente alinhados com a seleção, teste de métricas para avaliação da GC ou com a avaliação da GC nas organizações (estudos empíricos).

Ao final da etapa de seleção do portfólio bibliográfico obteve-se 11 artigos relevantes, alinhados com o tema e de reconhecimento científico. O portfólio pode ser observado na Figura 5.

Figura 5: Portfólio bibliográfico sobre avaliação de desempenho da gestão do conhecimento (mar/2011)

\begin{tabular}{|c|c|c|c|c|c|}
\hline$\#$ & Autor (Author) & Título (Title) & Periódico (Journal) & $\begin{array}{l}\text { Ano } \\
\text { (Year) }\end{array}$ & $\begin{array}{l}\text { Citações } \\
\text { (Citations) }\end{array}$ \\
\hline 1 & Ranjit Bose & Knowledge management metrics & $\begin{array}{l}\text { Industrial Management \& } \\
\text { Data Systems }\end{array}$ & 2004 & 110 \\
\hline 2 & $\begin{array}{l}\text { Pieris Chourides, David } \\
\text { Longbottom and William } \\
\text { Murphy }\end{array}$ & $\begin{array}{l}\text { Excellence in knowledge management: an } \\
\text { empirical study to identify critical factors and } \\
\text { performance measures }\end{array}$ & $\begin{array}{l}\text { Measuring Business } \\
\text { Excellence }\end{array}$ & 2003 & 74 \\
\hline 3 & $\begin{array}{l}\text { Marianne Gloet and Milé } \\
\text { Terziovski }\end{array}$ & $\begin{array}{l}\text { Exploring the relationship between knowledge } \\
\text { management practices and innovation } \\
\text { performance }\end{array}$ & $\begin{array}{l}\text { Journal of Manufacturing } \\
\text { Technology Management }\end{array}$ & 2004 & 59 \\
\hline 4 & $\begin{array}{l}\text { Mu-Yen Chen and An-Pin } \\
\text { Chen }\end{array}$ & $\begin{array}{l}\text { Knowledge management performance evaluation } \\
\text { A decade review from } 1995 \text { to } 2004\end{array}$ & $\begin{array}{l}\text { Journal of Information } \\
\text { Science }\end{array}$ & 2006 & 54 \\
\hline 5 & $\begin{array}{l}\text { Bernard Marr, Oliver Gupta, } \\
\text { Stephen Pike e Goran Roos }\end{array}$ & $\begin{array}{l}\text { Intellectual capital and knowledge management } \\
\text { effectiveness }\end{array}$ & Management Decision & 2003 & 50 \\
\hline 6 & $\begin{array}{l}\text { Bernard Marr, Gianni } \\
\text { Schiuma, Andy Neely }\end{array}$ & $\begin{array}{l}\text { Intellectual capital - defining key performance } \\
\text { indicators for organizational knowledge assets }\end{array}$ & $\begin{array}{l}\text { Business Process } \\
\text { Management Journal }\end{array}$ & 2004 & 49 \\
\hline 7 & $\begin{array}{l}\text { David P. Spicer, Eugene } \\
\text { Sadler-Smith }\end{array}$ & $\begin{array}{l}\text { Organizational Learning in Smaller } \\
\text { Manufacturing Firms }\end{array}$ & $\begin{array}{c}\text { International Small Business } \\
\text { Journal }\end{array}$ & 2006 & 33 \\
\hline 8 & $\begin{array}{l}\text { Michael Zack, James McKeen } \\
\text { and Satyendra Singh }\end{array}$ & $\begin{array}{l}\text { Knowledge management and organizational } \\
\text { performance: an exploratory analysis }\end{array}$ & $\begin{array}{l}\text { Journal of Knowledge } \\
\text { Management }\end{array}$ & 2009 & 17 \\
\hline 9 & $\begin{array}{l}\text { Kwong-Chi Lo and Kwai-Sang } \\
\text { Chin }\end{array}$ & $\begin{array}{l}\text { User-satisfaction-based knowledge management } \\
\text { performance measurement }\end{array}$ & $\begin{array}{l}\text { International Journal of } \\
\text { Quality and Reliability } \\
\text { Management }\end{array}$ & 2009 & 2 \\
\hline 10 & $\begin{array}{l}\text { C. Jyothibabu, Ayesha } \\
\text { Farooq, Bibhuti Bhusan } \\
\text { Pradhan }\end{array}$ & $\begin{array}{l}\text { An integrated scale for measuring an } \\
\text { organizational learning system }\end{array}$ & The Learning Organization & 2010 & 0 \\
\hline 11 & $\begin{array}{l}\text { Jae-Nam Lee and Byounggu } \\
\text { Choi }\end{array}$ & $\begin{array}{l}\text { Determinants of Knowledge Management } \\
\text { Assimilation: An Empirical Investigation }\end{array}$ & $\begin{array}{l}\text { Engineering Management, } \\
\text { IEEE Transactions on }\end{array}$ & 2010 & 0 \\
\hline
\end{tabular}

\subsection{Análise bibliométrica do portfólio bibliográfico}

Essa seção está dividida em três partes, cuja análise quantitativa dos artigos selecionados está organizada de acordo com a origem dos dados numéricos: portfólio, referências do portfólio e portfólio/referência.

\subsubsection{Análise dos artigos do portfólio}

Com a análise dos dados numéricos dos artigos selecionados busca-se avaliá-los quanto à relevância de seus periódicos, quanto ao reconhecimento científico, relevância dos autores e palavras-chave mais utilizadas. 
- Estimar grau de relevância dos periódicos: para os 11 artigos selecionados, conforme indicado na Figura 5, coluna "periódicos", nota-se que nenhum deles pertence ao mesmo periódico. Assim, para esse portfólio conclui-se que o assunto é de interesse de vários periódicos que trazem estudos relacionados à gestão;

- Estimar reconhecimento científico de artigos: na Figura 5 é possível avaliar, através do número de citação de cada um dos artigos selecionados, quais possuem maior reconhecimento científico. É importante também considerar a data de publicação, já que artigos recentes podem não possuir citações ainda que sejam de grande importância para o tema em estudo. $\mathrm{O}$ ano de publicação pode ser verificado também na Figura 5, coluna "Ano";

- Estimar o grau de relevância dos autores: conforme ilustrado na Figura 6, nota-se que apenas um dos autores dos artigos selecionados é autor de 2 artigos. Para a amostra considerada, essa também pode ser uma indicação de que a avaliação da gestão do conhecimento é de interesse de universidades e autores diversos.

Figura 6: Grau de relevância dos autores do portfólio bibliográfico

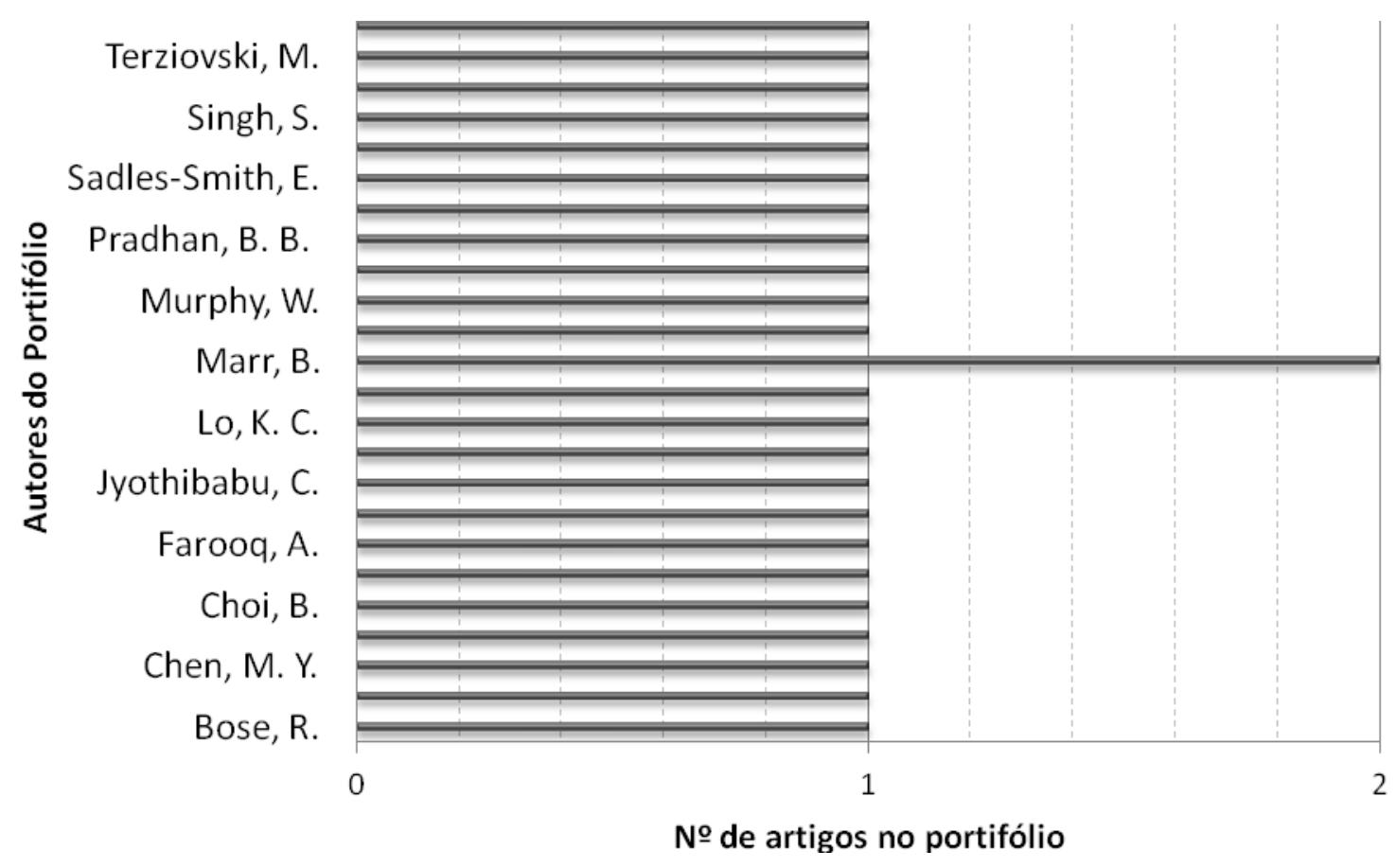

Fonte: Resultado da pesquisa (2011)

- Estimar palavras mais utilizadas: através da pesquisa das palavras-chave utilizadas por cada um dos 11 artigos do portfólio, é possível identificar aquelas que mais caracterizam o tema da pesquisa, Figura 7. Interessante observar que as palavras mais utilizadas também foram utilizadas por essa pesquisa para obter o resultado do item 4.1 .

\subsubsection{Análise das referências dos artigos do portfólio}


Nesta seção, os dados numéricos dos artigos serão analisados do ponto de vista de suas referências, isto é, para cada artigo do portfólio foram extraídas suas referências que se enquadravam como artigo publicado em periódico. Para os 11 artigos do portfólio foram encontradas 1020 referências do tipo artigo. A seguir, a análise dessas referências:

Figura 7: Palavras-chave dos artigos do portfólio

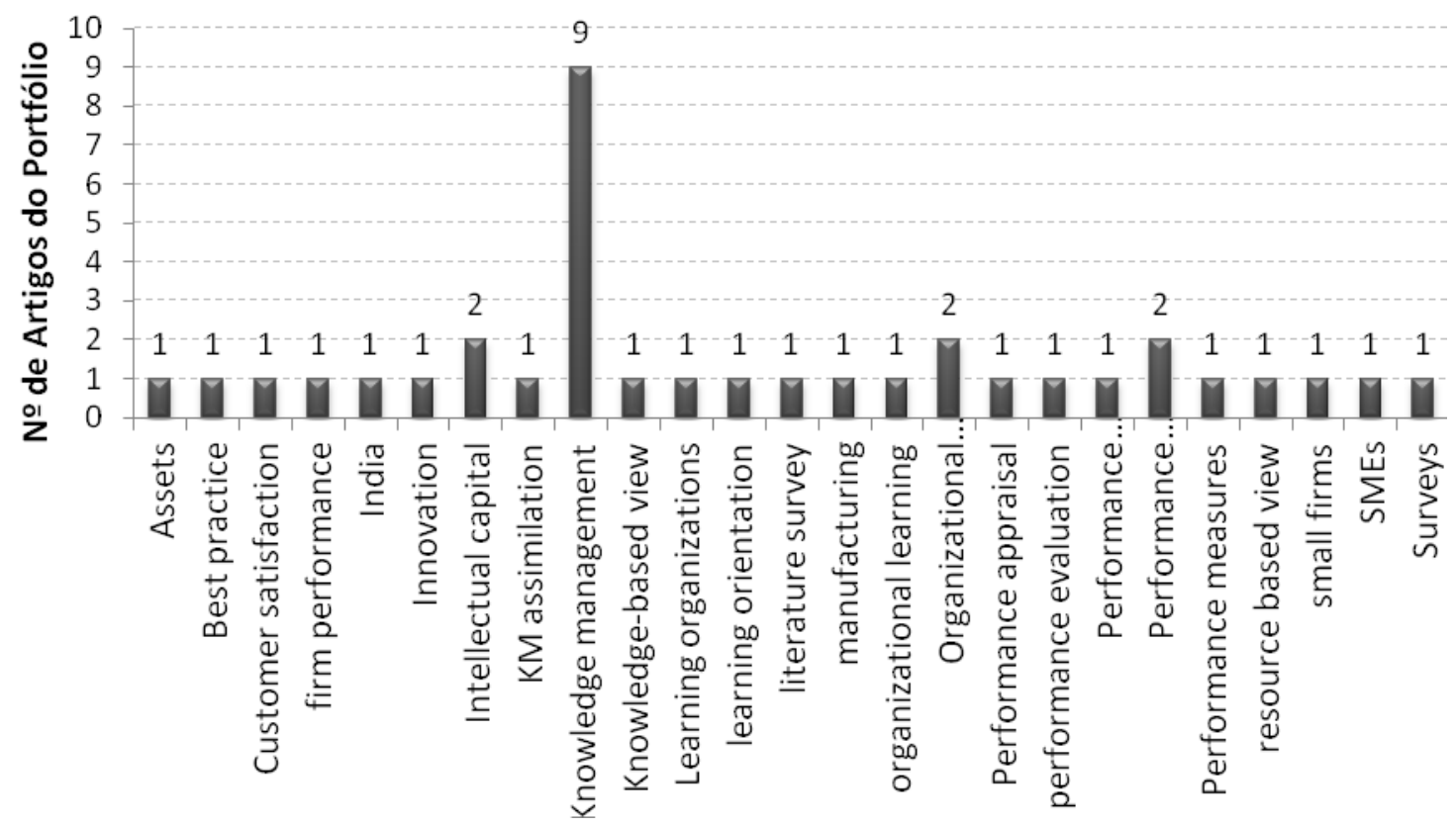

Fonte: Resultado da pesquisa (2011)

- Relevância dos periódicos das referências: as 1020 referências dos artigos do portfólio estão distribuídas em 344 periódicos diferentes. Na figura 8 pode-se observar os periódicos que mais se destacaram, isto é, que concentram $40 \%$ do número de referências encontradas. Em média, cada periódico teve 3 referências, um número relativamente baixo. Assim, pode-se inferir, para essa amostra, que a gestão do conhecimento é uma área de grande abrangência, de interesse de periódicos diferentes. 
Figura 8: Periódicos das referências que mais se destacaram (40\% do número de referências encontradas)
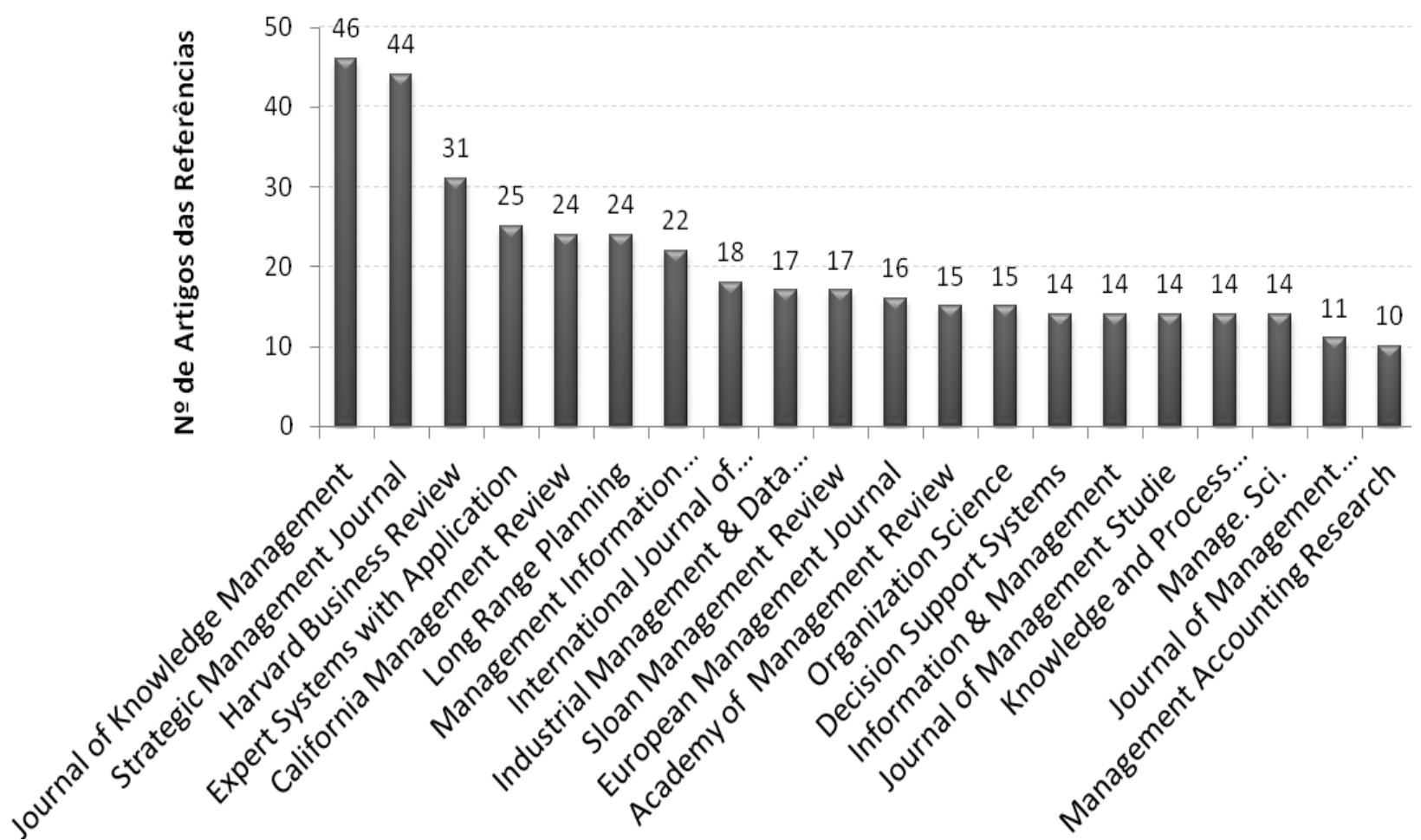

Fonte: Resultado da pesquisa (2011)

- Relevância dos autores das referências: para as 1020 referências foram identificados 1437 autores diferentes. Na Figura 9, podem ser observados os autores responsáveis por $10 \%$ do número de referências. É interessante observar que o autor mais citado nas referências pertence ao grupo de autores do portfólio. Essa relação pode ser observada na Figura 10.

Figura 9: Principais autores das referências.

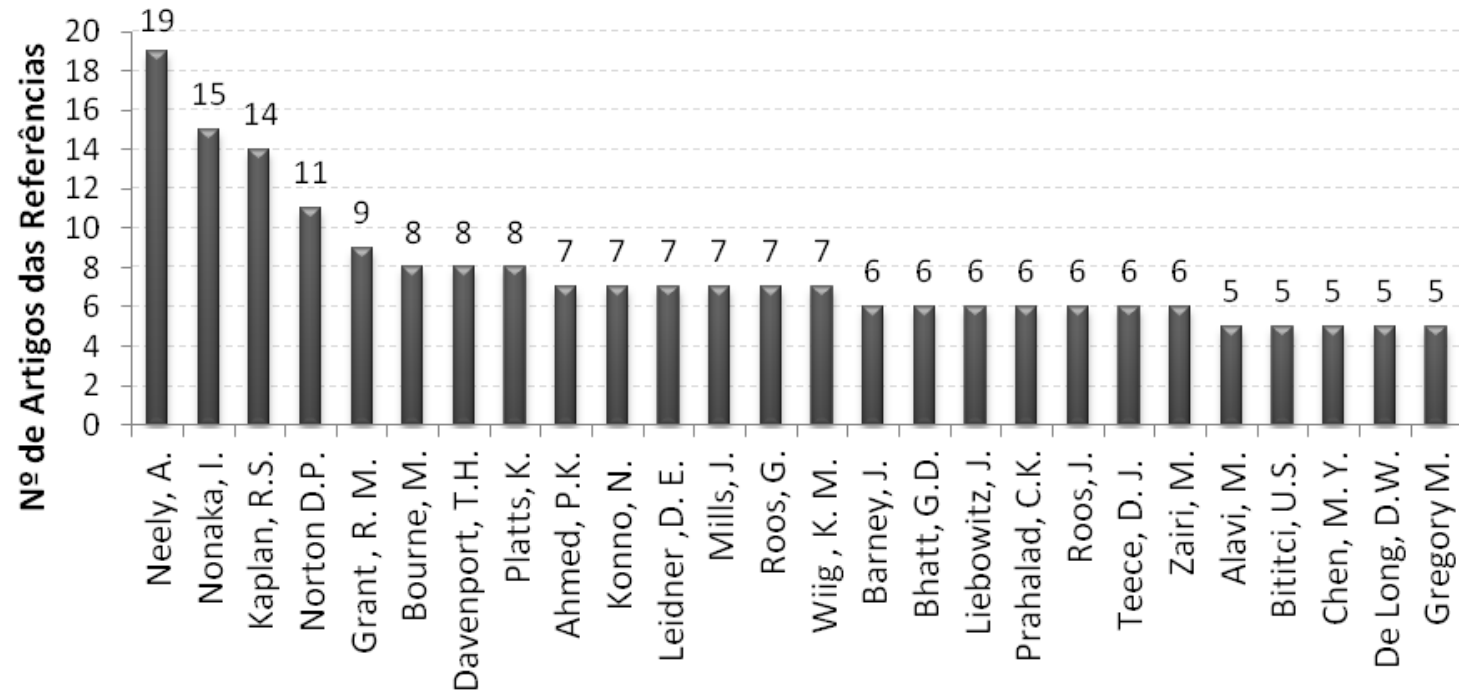

Principais Autores das Referências

Fonte: Resultado da pesquisa (2011) 
Figura 10: Quantidade de artigos das referências e do portfólio para cada autor do portfólio

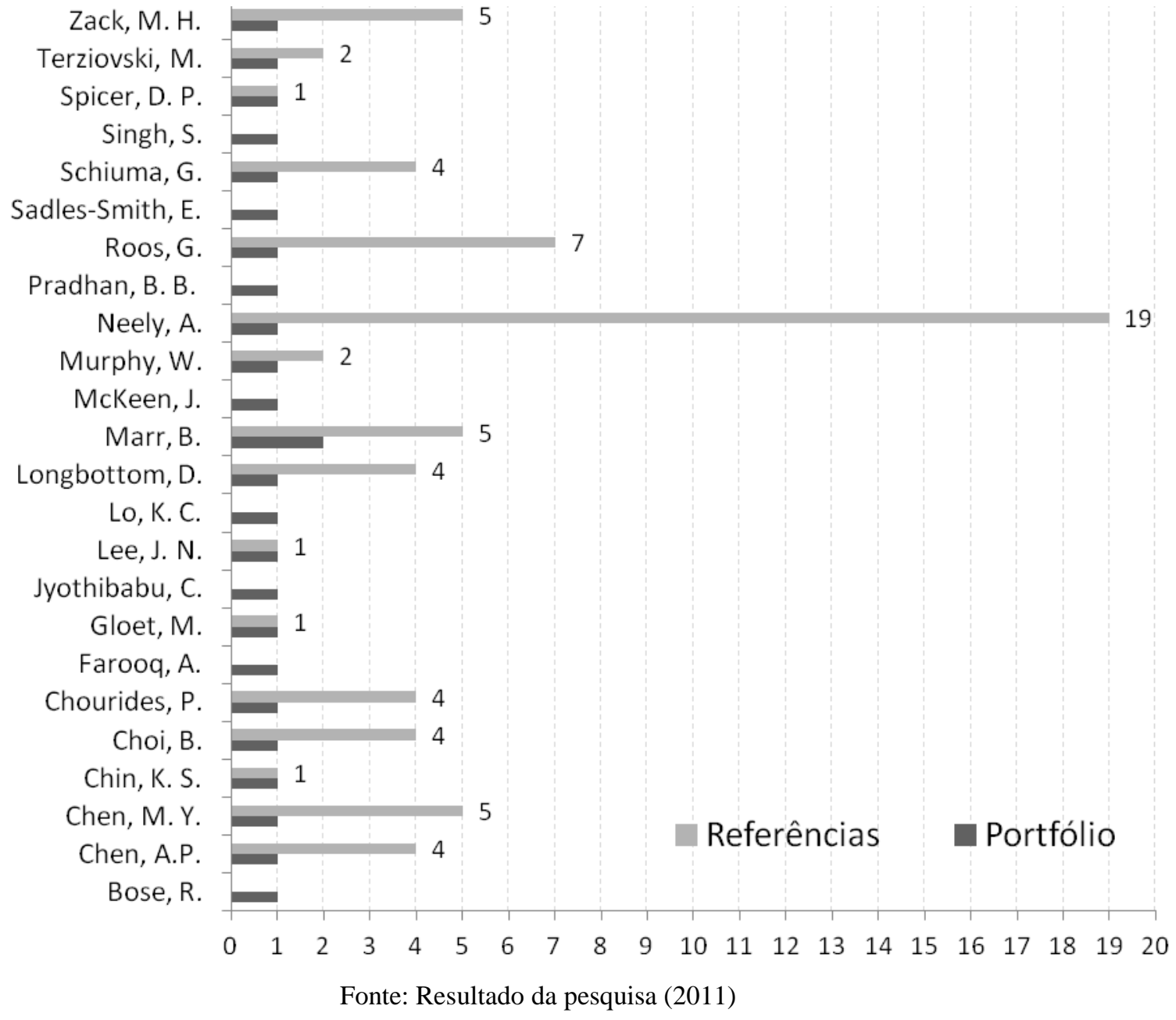

\subsubsection{Análise do artigos do portfólio mais suas referências}

Após a análise do portfólio e de suas referências cabe avaliar os artigos de maior destaque com relação à sua relevância acadêmica e aos autores mais citados nas referências. Para isso, o método ProKnow-C sugere a análise com relação às citações no Google Acadêmico e com relação ao número de citação dos autores do portfólio nas referências do portfólio, conforme visto no item 4.2.2. Assim, como podemos ver na Figura 11, existem 4 quadrantes de classificação dos artigos do portfólio. Nenhum artigo se enquadrou no grupo dos de maior destaque e produzido por autores de destaque. Por outro lado, os artigos 1, 2 e 3 (ver descrição dos artigos e autores na Fonte: Resultado da pesquisa (2011)

Figura 12) estão entre os que maior se destacaram por sua relevância acadêmica. Por fim, o artigo 6 se sobressai por ter sido produzido por autores de destaque. 
Figura 11: Artigos de destaque para o tema de pesquisa

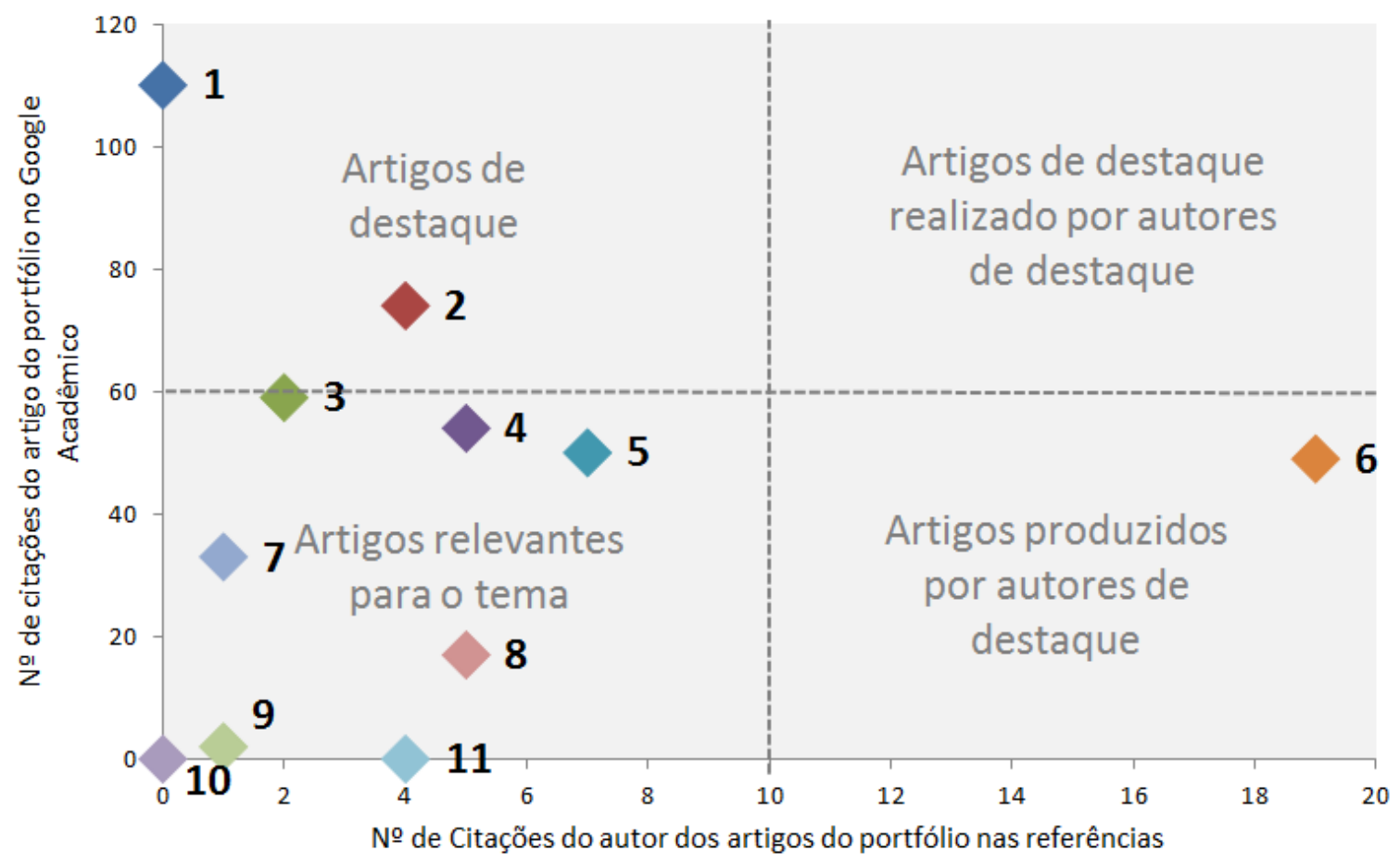

Fonte: Resultado da pesquisa (2011)

Figura 12: Descrição dos artigos de maior destaque para o tema de pesquisa

\begin{tabular}{|l|l|c|c|}
\hline \# & \multicolumn{1}{|c|}{ Autor (Author) } & \multicolumn{1}{c|}{ Título (Title) } & Ano (Year) \\
\hline $\mathbf{1}$ & Ranjit Bose & Knowledge management metrics & 2004 \\
\hline $\mathbf{2}$ & $\begin{array}{l}\text { Pieris Chourides, David Longbottom } \\
\text { and William Murphy }\end{array}$ & $\begin{array}{l}\text { Excellence in knowledge management: an empirical study } \\
\text { to identify critical factors and performance measures }\end{array}$ & 2003 \\
\hline $\mathbf{3}$ & Marianne Gloet and Milé Terziovski & $\begin{array}{l}\text { Exploring the relationship between knowledge } \\
\text { management practices and innovation performance }\end{array}$ & 2004 \\
\hline $\mathbf{6}$ & $\begin{array}{l}\text { Bernard Marr, Gianni Schiuma, Andy } \\
\text { Neely }\end{array}$ & $\begin{array}{l}\text { Intellectual capital - defining key performance indicators } \\
\text { for organizational knowledge assets }\end{array}$ & 2004 \\
\hline
\end{tabular}

\section{Considerações finais}

Esse estudo trouxe informações relevantes para interessados na pesquisa da gestão do conhecimento e sua performance. Os objetivos inicialmente propostos foram alcançados, isto é, obteve-se, ao final da aplicação do ProKnow-C, um portfólio de 11 artigos relevantes, de reconhecimento científico e alinhados com o tema de avaliação da gestão do conhecimento. Para chegar nos 11 artigos selecionados, definiu-se as palavras-chave em dois eixos de pesquisa, selecionou-se as bases de dados que mais estavam relacionadas como o tema, como também, fez-se um estudo quantitativo desse portfólio. O estudo bibliométrico, ou seleção dos artigos mais relevantes dos 11 obtidos, trouxe destaque para 4 artigos importantes para o tema. Um deles, 
principalmente, por que um de seus autores foi citado 19 vezes pelas referências dos artigos do portfólio.

O método utilizado de revisão da literatura, ProKnow-C da UFSC, permite sistematizar essa atividade, embora ainda seja mantida certa subjetividade na seleção dos artigos no que diz respeito ao alinhamento com o tema de pesquisa. Essa subjetividade, no entanto, faz parte do processo, uma vez que cada indivíduo possui seu próprio interesse e objetivos sobre determinado tema de pesquisa. Com relação à aplicação do método para o tema de avaliação de desempenho da gestão do conhecimento, é possível concluir, pelos resultados numéricos obtidos e o nível de relevância dos artigos selecionados, que se trata de uma ferramenta de grande importância acadêmica. A importância é devido à sistematização desse tipo de atividade necessária para a maioria dos tipos de pesquisa. Além disso, a sistematização simplifica e organiza as tarefas de pesquisa, gerando, como resultado, a ampliação do conhecimento do pesquisador sobre o tema desejado assim como meios de justificativa das referências utilizadas na pesquisa.

Dos resultados obtidos, a identificação dos autores de destaque, cujas leituras sejam significativas para o tema é o ponto inicial para qualquer tipo de pesquisa. Conhecer os periódicos que mais publicam sobre o tema de pesquisa indica, também, os periódicos mais suscetíveis a aceitar a publicação gerada a partir dessa pesquisa.

Autores como Bose (2004), Chen e Chen (2006), Igarashi (2010) também realizam pesquisas na literatura, no entanto, não fica claramente evidenciado o método utilizado de seleção de portfólio assim como sua quantificação. O ProKnow-C facilita a justificativa de seleção de portfólios para pesquisas de revisão da literatura ou que utilizam a revisão da literatura como ponto de partida para estudos empíricos, por exemplo. A limitação do portfólio selecionado através desse método está na seleção de artigos escritos somente na lingua inglesa, já que publicações em outros idiomas também podem ser de grande relevância para o tema.

\section{Abstract}

The aim of this study was to apply the method ProKnow-C, developed by MCDA Laboratory, of Federal University of Santa Catarina, to research and analyze quantitatively the articles with scientific prestige and relevants to the topic of Knowledge Management from the perspective of performance evaluation. The method used it is the systematic process of searching and selecting articles proposed by ProKnow-C, making use of bibliometric analysis techniques and also considering what the researcher deems relevant to the research topic. The main result has been the validation of the method used to develop knowledge on the subject searched. Not least, the method allowed to obtain a bibliographic portfolio relevant to literature and with scientific prestige. Details such as the main authors and main keywords used for the subject, are of interest to researchers. Eventually, the result obtained in this research by applying the method can contribute to studies whose interest lies in the evolution in time of the theme performance evaluation of knowledge management. 
Keywords: bibliometrics; literature review; performance evaluation; knowledge management.

\section{Referências}

AFONSO, M. H. F. et al. Como construir conhecimento sobre o tema de pesquisa? Aplicação do processo ProKnow-C na busca de literatura sobre avaliação do desenvolvimento sustentável. RGSA: Revista de Gestão Social e Ambiental, v.5 pp. 47-62, 2012.

ARAUJO, C. Bibliometria: evolução histórica e questões atuais. Em Questão, v. 12, pp. 11-32, 2006.

BOSE, R. Knowledge Management Metrics. Industrial Management \& Data System, n. 104, pp. 457-468, 2004.

\section{cross ref}

CAPES. Portal de Periódicos. Disponível em: <http://www.periodicos.capes.gov.br>. Acesso em: mar 2011.

CAUCHICK et al. (2010) Metodologia de pesquisa em engenharia de produção e gestão de operações. Rio de Janeiro: Elsevier.

CHEN, M.-Y.; CHEN, A.-P. Knowledge management performance evaluations: a decade review from, 1995 to 2004. Journal of Information Science, v. 32, pp. 17-38, 2006.

\section{cross $r$}

CHOURIDES, P.; LONGBOTTOM, D.; MURPHY, W. Excellence in Knowledge Management: an empirical study to identify critical factors and performance measures. Measuring Business Excellence, v. 7, pp. 29-45, 2006.

cross ref

DAVENPORT, T.; DE LONG, D.; BEERS, M. Successful knowledge management projects. Sloan Management Review, 39, pp. 43-57, 1998.

ENSSLIN, L et al. ProKnow-C: Processo de análise sistêmica. Brasil: Processo técnico com patente de registro pendente junto ao INPI, 2010.

GLOET, M.; TERZIOVSKI, M. Exploring the relationship between knowledge management pratices and innovation performance. Journal of Manufacturing Technology Management, v. 15, pp. 402-409, 2004.

\section{cross ref}

GOOGLE ACADÊMICO. Disponível em <http://scholar.google.com.br>. Acesso em: mar 2011.

IGARASHI, W. Aprendizagem organizacional: proposta de um modelo de avaliação. Florianópolis: Universidade Fedreal de Santa Catarina, 2009.

MORETTI, S. L. A.; CAMPANARIO, M. A. A produção intelectual brasileira em Responsabilidade Social Empresarial - RSE sob a ótica da bibliometria. RAC, v.13, pp. 68-86, 2009.

NARIN, F. Evaluative bibliometrics: the use of publication and citation analysis in the evaluation of scientific activity. Cherry Hill: Computer Horizons Inc, 1976.

OLIVEIRA, E. F. T.; GRACIO, M. C. C.. Indicadores bibliométricos em ciência da informação: análise dos pesquisadores mais produtivos no tema estudos métricos na base Scopus. Perspectivas em Ciência da Informação, v. 16; n.4, pp. 16-28, 2011.

PABLO, P. A guideline for building an intellectual capital statement: the 3R model. Int. J. Learning and Intellectual Capital, v. 1, pp. 3-18, 2004. 
SPICER, D. P., SADLER-SMITH, E. Organization Learning in Smaller Manufacturing Firms. International Small Business Journal, v. 24, n. 2, pp 133-158, 2006.

\section{cross ref}

TASCA, J. E. et al. An approach for selecting a theoretical framework for the evaluation of training programs. Journal of European Industrial Training, v. 34, pp. 631-655, 2010.

\section{cross ref}

TERRA, J. Gestão do Conhecimento: o grande desafio empresarial. São Paulo: Negócio Editora, 2005.

\section{Dados dos autores:}

Nome completo: Lílian de Oliveira Vilela

Filiação institucional: Universidade Federal de Santa Catarina - UFSC

Departamento: Departamento de Engenharia de Produção e Sistemas

Função ou cargo ocupado: Mestranda em Eng ${ }^{a}$ de Produção

Endereço completo para correspondência (bairro, cidade, estado, país e CEP): Campus Universitário, Trindade, Florianópolis, SC, Brasil - 88040-900

Telefones para contato: (47) 9105-9636

e-mail: lilian.oliveira.vilela@gmail.com

\section{Enviado em: 22/06/2011}

